\title{
GLIOMA OF THE RIGHT OPTIC THALAMUS AND CORPORA QUADRIGEMINA.
}

\author{
BY DAVID FERRIER, M.D., F.R.S., \&c. \\ Physioian to the National Hospital for the Paralysed and Epiloptio.
}

TuE following case seems worthy of record, as illustrating the exceedingly complex symptomatology of tumours affecting the tegmentum and its ganglia.

Samuel H., æt. 14, was admitted into the National Hospital for the Paralysed and Epileptic on May 13, 1881.

Previous History.-The commencement of the present illness was thought to be a fall on the ice four years ago. Some kind of fit occurred the day after, but there were no other symptoms till September 1880. In this month he had a fit. In October he har a series of fits, with general convulsions and retraction of the head, lasting for about five hours. Next day he had frequent vomiting. In November he had two fits of a similar character. It was observed that after these fits the right eye was turned inwards, and continued so more or less for six weeks. At this time there was diplopia. Several fits occurred, and occasional sickness subsequently, but he was apparently improving and getting stronger, till a month before admission, when it was observed that he began to reel in his gait, and to use the left leg with difficulty. There seemed to be a tendency to reel specially towards the left side. This difficulty in walking had gone on increasing, so that he had not been able to walk alone for a fortnight.

The left arm was also observed to be affected, being unsteady and weak, so that the patient conld not use his left hand to button his clothes or hold a fork.

The patient had occasional attacks of hesdache, and also pain and numbness in the left arm.

State on admission. - Exceedingly dull and heavy-looking, but responds intelligently to questions as to name, age, \&c. Says he has pain in the back of the head on pressure or percussion.

Motility.-The ocular movements upwards and downwards are normal, but the lateral sweep is restricted, especially towards the left and a nystagmus tends to occur. 
The power of converging the eyes is entirely lost, so that he cannot look at the tip of his nose or at an object brought close up to the eyes. Nor do the pupils show any contraction at the attempts at accommodation. The movements of the face, tongue and jaws are apparently unaffected. The arms retain all their movements, but the left is distinctly weaker than the right. Grasp of right hand $=45 \mathrm{lbs}$, of left $=15 \mathrm{lbs}$.

The left hand also oscillates when he attempts to touch or lay hold of anything. Otherwise it remains still and free from all tremors or twitching.

The legs can be moved freely in all directions when he is lying in bed, though the left is distinctly foebler than the right. In attempting to walk he lifts the left foot high off the ground, and throws it forward in a brusque and ataxic manner. Cannot walk withont support.

The patellar reactions are very distinct, the left excessive and almost tetanic. Ankle-clonus is readily induced in the left. The plantar, abdominal and epigastric reflexes are obtained on both sides, but better on the right side. The cremasteric reflex was obtained equally on both sides.

Sensibility. - Tactile sensibility is somewhat defective on the whole of the left side, but painfal impressions are felt and correctly localised.

Sight is good. He can read 3 Nettleship with the right eye, and 4 Nettleship with the left.

The pupils are equal and react to light, but there is no alteration on attempts at accommodation. Ophthalmoscopic examination reveals well-marked double optic neuritis.

Hearing is better on the right than left side. Hears a watch at 18 in. on left, at 3 foot on right.

Taste and smell good, and no appreciable difference on the two sides.

The subsequent history may be summed up shortly as increasing torpor and mental hebetude, greater weakness, amounting towards the end to complete paralysis. of the left arm and leg, and partial of the face, with anæsthesia and analgesia. Fits occurred at intervals, varying somewhat in character, but all tonic rather than clonic.

In one, in which he was carefully observed by the attendent, both arms were rigidly flexed, the legs Hexed at the knees, the face drawn to the right, the head retracted, and both eyes widely opened and turned upwards. The spasms lasted about a minute, and were followed by unconsciousness for several hours.

In another attack the arms were rigidly extended, the hands clenched, the head retracted, and the legs extended.

In other attecks, again, there was loss of consciousness with- 
out muscular rigidity. In one of these, seen by Dr. Beevor, he lay with the body turned to the left. The face was dusky and covered with perspiration. The right pupil was somewhat linger than left, and insensible to light, while the left reacted slightly. Slight external strabismus existed in the right eye. The breathing was slow, and the pulse sixty per minute. At the end of ten minutes he opened the eyes and moved the right limbs a little. Well-marked ankle-clonus and kneojerk existed in the right. Plantar reflex was obtained on the right side slightly, but none on the left.

Death occurred on July 27 th, 1881.

\section{Post-Mortem Examination.}

The skull cap was thin. The dura mater was not adherent, but tense. The convolutions were somewhat flattened and dry, the veins being large and distended. The right parietal region felt firmer than the rest of the brain.

The cranial nerves were intact.

The posterior extremity of the right optic thalamus projected behind the crus cerebri and was in contact with the middle peduncle of the cerebellum,-as seen from the base.

On division of the corpus callosum and exposure of the lateral ventricles and corpora quadrigemina, the right optio thalamus and right side of the corpora quadrigemina appeared, while retaining their normal shape and surface, larger than the left.

The greatest antero-posterior diameter of the right thalamus was 2 inches, while that of the left was $1 \frac{1}{2}$ inch. The greatest width of the right was 2 inches, but of the left only inch.

'The right anterior tubercle of the corpora quadrigemina was nbout $t$ inch larger in all its diameters than the left. The consistence of the enlarged parts wes softer than the normal structure, and, on section, the surface presented the characteristic appearances of a glioma shading imperceptibly into the surrounding tissue. The internal cupsule and ganglionio structure of the right thalamus and lenticular nucleus were indistinguishable, and the sub-thalamic region and tegmentum of the crus cerebri seemed involved in the growth. The foot of the crus cerebri and locus niger were distinct. The nucleus caudatus, internal capsule and anterior part of the lenticular nucleus were of normal appearance. The enlarged optic thalamus pressed backward against the anterior or quadrangular lobe of the right side of the cerebellum, and was closely appressed and moulded against this and the middle peduncle. 'Ilis portion of the cerebellum, instead of having the laminne running parallel to euch other, forward and outwards, had an 
irregularly convoluted appearance, as if thrown into folds by compression into a smaller space. The pressure on the anterior surface of the right lateral lobe caused the inferior semilunar lobe to be closely applied against the medulla oblongatir. The right lateral lobe of the cerebellum was thus somewhat distorted, and the antero-posterior diameter shortened by about half-an-inch as compared with the left.

Remarks. - The dingnosis made in this case, viz., a tumour affecting the right side of the cerebellum and pons, was only approximately correct, nor does it seem possible, even with the actual condition before us, to infer otherwise than vaguely as to the symptoms which should have existed during life.

The fact of the existence of an intra-cranial tumour was indicated by the general symptoms, viz., headache, romiting, optic neuritis and convulsions : and the reeling gait and tonic or tetanic nature of the convulsive attacks were symptoms which, as has been pointed out by Hughlings-Jackson, aro characteristic of tumours of the cerebellum. That the cerebellum was affected here is very clearly shown by the compression and distortion of the right lobe by the backward grotwth of the tumour; but whether in this, or in the cases where the tumour is actually in the cerebellum itself, the tonic spasms are in reality cerebellar, or, as I am more inclined to regard them, due to irritation of the corpora quadrigemina, is a point not to be decided by clinical facts alone. But the facts of experimental irritation of the cerebellum and of the corpora quadrigemina, which I have elsewhere described, would lend me to regard the affection of the corpora quadrigemina as the essential factor. In this case the corpora quadrigemina were directly implicated by the invasion of the morbid growth, and several of the symptoms seen in this case have been found in connection with tumours in this neighbourhood. Disorders of locomotion, and certain forms of ophthalmoplegia have been specially noted. In this case there was total paralysis of the convergence of the eyes, and weakness of the lateral movements, the others being unaffected. But there was no paralysis of reflex irido-motor action, and there was scarcely any appreciable difference in the power of vision in the two eyes. The right was a trifle more acute than the left. The locomotor incoordination was very marked, not merely disorder of equilibration, but a curious ataxic action of the legs. Cases of impaired co-ordination of locomotion with ophthalmoplegia in connection with indications pointing to disease in this region have important physiological bearings. The partial hemiplegia of motion and sensation on the left side, which might have been the result of a tumonr of the right half of the cerebellum pressing on the subjacent 
sensory and motor tracts, was no doubt, in this case, due to the direct implication of the right intermal capsule which was involved in the growth. The oscillations of the left hand on volitional movements may be regarded also as due to the same cause, in accordance with the observations of Charcot in reference to the lesion in post-hemiplegic choreic disorders. 